\title{
Comparison of successful versus failed percutaneous coronary intervention in patients with chronic total occlusion: A systematic review and meta-analysis
}

\author{
Dongfeng Zhang, Nan Nan, Yuguo Xue, Mingduo Zhang, Jinfan Tian, \\ Changzhe Chen, Min Zhang, Xiantao Song \\ Department of Cardiology, Beijing Anzhen Hospital, Capital Medical University, \\ Beijing Institute of Heart Lung and Blood Vessel Disease, Beijing, China
}

\begin{abstract}
Background: The optimal treatment strategy of chronic total occlusion (CTO) is currently debated. This meta-analysis aimed to evaluate the long-term clinical outcomes of successful percutaneous coronary intervention (PCI) of CTO.

Methods: Electronic databases were searched for studies comparing long-term outcomes between successful PCI in patients with CTO using drug-eluting stents and failed procedures. Meta-analysis was conducted with major adverse cardiac events (MACE) and all-cause mortality during the longest follow-up as endpoints. The combined hazard ratios (HRs) were applied to assess the correlation between successful CTO PCI and MACE/all-cause mortality.

Results: Eight studies consisting of 6,211 patients published between 2012 and 2020 met our inclusion criteria, and the CTO PCI success rate was $81.2 \%$. Patients in the failed group were much older, and more likely to have morbidities (hypertension and prior myocardial infarction), reduced left ventricular ejection fraction, and severe lesion characteristics (multivessel disease and moderate/severe calcification). Pooled results indicated that successful CTO PCI was significantly associated with prognosis. Compared to failed recanalization, patients receiving successful procedures had an improved MACE (hazard ratio [HR]: 0.50, 95\% confidence interval [CI]: 0.40-0.61, $p<0.001$ ). Subgroup analyses further revealed the prognostic value of successful CTO PCI. However, no difference was observed regarding all-cause mortality (HR: 0.79, 95\% CI: 0.61-1.02, $p=0.074$ ).

Conclusions: The present study showed that CTO recanalization was associated with improved long-term outcomes. However, randomized trials are needed to confirm the results due to the mismatch of baseline characteristics. (Cardiol J)
\end{abstract}

Key words: chronic total occlusion, percutaneous coronary intervention, major adverse cardiac events, meta-analysis

\section{Introduction}

According to the coronary Chronic Total Occlusion Academic Research Consortium (CTO-ARC) consensus recommendations, definite coronary chronic total occlusion (CTO) indicates CTO with typical appearance and definitive corroborating evidence of occlusion duration $\geq 3$ months [1]. Typical

Address for correspondence: Dr. Xiantao Song, Department of Cardiology, Beijing Anzhen Hospital, Capital Medical University, Beijing Institute of Heart Lung and Blood Vessel Disease, 2 Anzhen Road, Chaoyang District, Beijing, 100029, China, tel: +86 10 64456371, fax: +86 10 64456470, e-mail: xiantao_song@163.com

Received: 22.04.2021 Accepted: 8.02.2022 Early publication date: 18.02.2022

This article is available in open access under Creative Common Attribution-Non-Commercial-No Derivatives 4.0 International (CC BY-NC-ND 4.0) license, allowing to download articles and share them with others as long as they credit the authors and the publisher, but without permission to change them in any way or use them commercially. 
appearance included Thrombolysis in Myocardial Infarction (TIMI) grade 0 flow through the lesion with no thrombus, no staining at the proximal cap, and presence of mature collaterals. CTOs are highly prevalent among patients undergoing diagnostic coronary angiography, ranging from one-quarter to one-third of patients, though the prevalence is related to the group studied [2-6]. Patients with a history of coronary artery bypass graft (CABG) surgery are found to have CTOs of their native vessels more frequently (54\%) [3], while patients presenting with ST-segment elevation myocardial infarction (STEMI) are less likely to have a CTO (10\%) [7].

Chronic total occlusion was once treated as the last frontier of interventional cardiology for low success rates and potential for increased complications. In the past two decades, the rate of successful percutaneous coronary intervention (PCI) has steadily increased due to the development of equipment, progression of technology, and accumulation of operation experience. The 2011 ACCF/AHA/SCAI PCI guidelines recommend PCI of CTO in patients with appropriate clinical indications and suitable anatomy when performed by operators with appropriate expertise (Class IIa, level of evidence [LOE] B) [8]. As recommended by the ESC/EACTS guidelines on myocardial revascularization, percutaneous revascularization of CTOs should be considered in patients with angina resistant to medical therapy or with a large area of documented ischemia in the territory of the occluded vessel (Class IIa, LOE B) [9].

Although success rates for recanalization of CTO continue to improve, the optimal treatment strategy remains debatable. This meta-analysis was performed to compare long-term clinical outcomes of successful PCI using drug eluting stent (DES) versus failed PCI in patients with CTO.

The following article is presented in accordance with the Preferred Reporting Items for Systematic Reviews and Meta-analyses (PRISMA) reporting checklist.

\section{Methods}

\section{Search strategy}

A systematic search was conducted for eligible studies published in English in PubMed, MEDLINE, and Cochrane databases before July 2020. Search terms used include: "chronic total occlusion", "coronary occlusion", "percutaneous coronary intervention", and "recanalization". Additionally, the cited articles of the included studies and related reviews with the same topic were screened by the two authors of this study (M.Z. and M.D.Z.).

\section{Inclusion criteria}

Both prospective and retrospective studies were eligible for further evaluation. All of the CTO definitions were consistent with the CTO-ARC standard. For inclusion, studies needed to: focus on patients with single or multiple CTO with attempted PCI, have a patient population divided into successful and failed PCI groups, provide endpoint data of interest beyond 1 year, have adjusted hazard ratios (HRs) and 95\% confidence intervals (CIs) for the endpoints available, and use DES only. Studies that exclusively enrolled patients with acute myocardial infarction (MI) were excluded.

Additionally, unpublished data or conference abstracts were not considered for inclusion. All eligible studies for inclusion were reviewed to identify overlapping of the study population, and in these cases the most recent study with the largest sample size was used. Disagreements during article evaluation were resolved through discussion with another researcher (D.F.Z.).

\section{Data extraction}

The following information was collected from the included studies by two independent researchers (N.N. and Y.G.X.): the first author's name, year of publication, study design, patient characteristics, intervention variables, and endpoints. For articles in which interested endpoints were not provided, emails were sent to the primary author for detailed information. A consultation regarding differences in data extraction was held with another researcher (D.F.Z.). Quality of the included studies was assessed using the Newcastle-Ottawa Scale. A study could be awarded a maximum of 9 stars for quality, and studies with a score greater than 7 were considered to be of high quality. Each study was assessed independently by 2 investigators (J.F.T. and C.Z.C.).

\section{Endpoints}

Most of the studies defined success CTO PCI as technical success, which was defined as achievement of TIMI grade 2 or greater antegrade flow in all $\geq 2.5 \mathrm{~mm}$ distal branches with $<30 \%$ residual stenosis of the target CTO lesion at the end of the procedure. Park et al. [10] defined as success of CTO revascularization procedure using DES implantation without serious procedure-related in-hospital deaths or major adverse events.

The following endpoints were collected from the eligible studies: the primary endpoint of major 


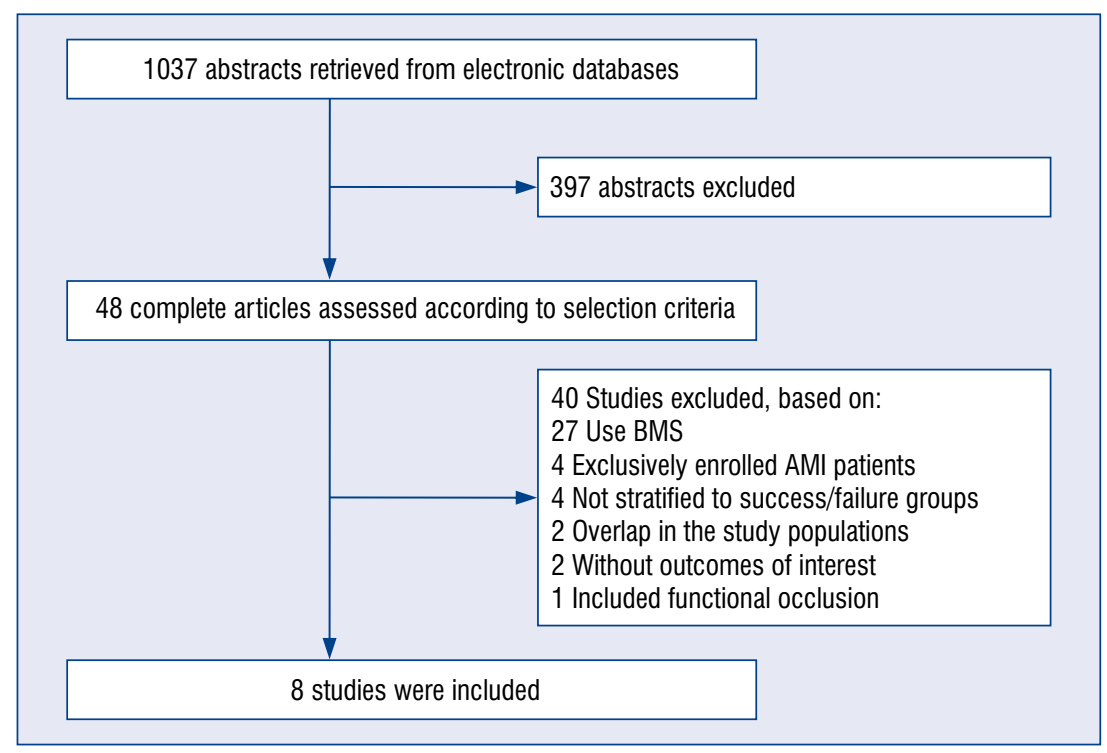

Figure 1. Flow diagram of study inclusion and exclusion criteria; $\mathrm{AMI}$ - acute myocardial infarction.

adverse cardiac events (MACE) and secondary endpoint of mortality from any cause during the longest follow-up. Follow-up periods were as reported in each study, which ranged from 1 year to 7 years. The definitions of MACE were also as those indicated in the individual studies. Most of the definitions, except the one carried by Park et al. [10] were the composite of death, MI, and targetvessel revascularization. Park et al. [10] defined MACE as all cause death or MI.

\section{Statistical analysis}

All statistical analyses were carried out using Stata 11.0 (Stata Corp, College Station, TX). The mean of continuous variables and percentage (\%) of categorical variables were calculated. The associations between successful CTO PCI and endpoints were expressed as the HRs with their corresponding 95\% CIs. Heterogeneity across studies were evaluated by Cochran's Q test and Higgins' ${ }^{2}$ statistic. The fixed effect model was adopted for nonsignificant heterogeneity $\left(\mathrm{I}^{2}<50 \%\right.$, $\mathrm{p}>0.1$ ). Publication bias was assessed by the visible plot and Begg's test. Sensitivity analysis was performed for the measurement of the reliability of the combined results.

\section{Results}

\section{Search results}

A literature search identified 7 prospective trials and 1 retrospective trial published between 2012 and 2020 [10-17] which provided the HRs and
95\% CIs in multivariate analysis for at least one of the endpoints, including 7 studies for MACE and 3 studies for all-cause mortality. If a study considered patients with failed CTO PCI as the reference, then the data was converted to HR estimations considering cases with successful CTO PCI as a reference group to reflect the impact of successful CTO PCI on CTO patients.

A total of 6,211 patients were included in these 8 studies. Figure 1 shows the inclusion and exclusion processes during the literature search process. Of these were 4 from Europe, 3 from Asia, and 1 from North America. Five were single-center experiences. The study characteristics and quality assessment results are shown in Table 1.

The final analysis comprised 5,044 patients who underwent successful recanalization of CTO lesions using DES and 1,167 patients with failed percutaneous interventions. The success rate of CTO PCI was $81.2 \%$. Supplementary Table 1 provides the demographic data, medical histories, and clinical characteristics of the included patients. In addition, summaries of variable means/percentages were calculated for the overall patient population. Compared with the successful recanalization patients, subjects in the failed group were much older (64.7 vs. 62.7 years, $p<0.001$ ), more likely to suffer from hypertension $(77.9 \%$ vs. $76.2 \%$, $\mathrm{p}=0.004)$, more likely to have a history of MI (37.5\% vs. $29.9 \%, \mathrm{p}<0.001)$, have a left ventricular ejection fraction (LVEF) of $\leq 40 \%(15.8 \%$ vs. $11.9 \%, \mathrm{p}=0.03$ ), have multivessel disease (MVD) $(75.0 \%$ vs. $67.9 \%, \mathrm{p}<0.001)$, and have 


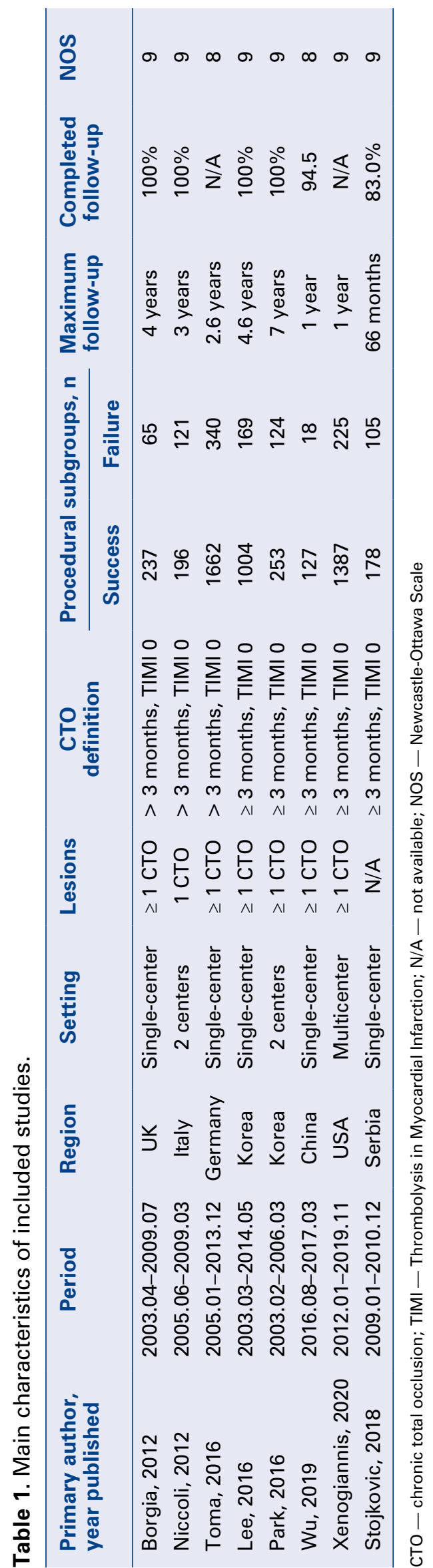

moderate-to-severe calcification $(61.7 \%$ vs. $46.2 \%$, $\mathrm{p}<0.001$ ). No differences were observed between successful and failed recanalization for male sex ( $82.8 \%$ vs. $84.2 \%, p=0.13)$, current smokers $(24.6 \%$ vs. $24.6 \%, \mathrm{p}=0.19)$, diabetes $(33.7 \%$ vs. $33.1 \%, \mathrm{p}=0.60)$ or hypercholesterolemia $(62.4 \%$ vs. $62.0 \%, \mathrm{p}=0.59)$.

Newcastle-Ottawa Scale quality assessment for the included studies indicated that all studies were of high quality. No publication bias for the included trials was observed.

\section{Main outcomes}

In summary, 7 studies with a total of 4,209 definite CTO patients reported the HRs for the association between successful recanalization of CTO lesions and MACE. Heterogeneity was not observed with an $\mathrm{I}^{2}$ statistic of $41.7 \%$, and the fixed effects model was selected. The pooled analysis showed that compared with failed CTO PCI, successful CTO PCI had a significantly lower MACE (HR: 0.50, 95\% CI: 0.40-0.61, p < 0.001), which indicates that the successful recanalization of CTO lesions may decrease the risk of MACE (Fig. 2).

The prognostic values of successful CTO PCI were further displayed in subgroup analyses. The setting (single-center vs. multicenter) and followup time ( $\leq 3$ years vs. $>3$ years) did not affect the significant association between successful CTO PCI and improved MACE (Fig. 3).

Three studies with 3,552 subjects assessed the association between successful CTO PCI and all-cause mortality. No heterogeneity was observed across studies according to the $\mathrm{I}^{2}$ statistic $(0 \%)$ thus, the fixed effects model was selected. No significant difference was found in the combined results of long-term death of any cause (HR: 0.79, 95\% CI: 0.61-1.02, $\mathrm{p}=0.074$ ) (Fig. 4).

\section{Sensitivity analysis}

Sensitivity analyzes conducted by excluding one study at a time showed the results of the present analyzes were relatively stable in this meta-analysis (Fig. 5).

\section{Discussion}

The present meta-analysis showed that patients receiving successful PCI for CTO lesions using DES suffered less from MACE than those who underwent failed procedures. However, no advantage was observed with regard to all-cause mortality. However, the results should be interpreted with a view of certain bias. Patients with 


\begin{tabular}{|c|c|c|}
\hline $\begin{array}{l}\text { Study } \\
\text { ID }\end{array}$ & $\mathrm{HR}(95 \% \mathrm{Cl})$ & $\begin{array}{c}\% \\
\text { Weight }\end{array}$ \\
\hline Borgia 2012 & $0.30(0.13,0.68)$ & 6.50 \\
\hline Niccoli 2012 & $0.55(0.24,0.75)$ & 13.71 \\
\hline Lee 2016 & $0.42(0.29,0.60)$ & 33.68 \\
\hline Park 2016 & $0.90(0.46,1.79)$ & 9.64 \\
\hline Stojkovic 2018 & $0.40(0.20,0.82)$ & 8.94 \\
\hline Wu 2019 & $0.17(0.05,0.59)$ & 2.92 \\
\hline Xenogiannis 2020 & $0.66(0.44,1.03)$ & 24.61 \\
\hline Overall $\left(I^{2}=41.7 \%, p=0.113\right)$ & $0.50(0.40,0.61)$ & 100.00 \\
\hline 0.05 & 20 & \\
\hline
\end{tabular}

Figure 2. Forest plot for the correlation between successful chronic total occlusion percutaneous coronary intervention and major adverse cardiac events; $\mathrm{HR}$ - hazard ratio; $\mathrm{Cl}$ - confidence interval.

failed intervention were much older, and more likely to have morbidities (hypertension and prior MI), reduced LVEF, and severe lesion characteristics (MVD and moderate/severe calcification), which have been shown to be poor prognostic factors in patients undergoing PCI. It is possible that the results represent the less favorable clinical profiles of patients with failed CTO PCI rather than the beneficial effects of successful CTO PCI. The worse outcome of the failed group might be partially due to a more severe coronary heart disease or to the resulted produced by the attempt of intervention.

Chronic total occlusion implies total occlusion of coronary arteries and is related to worse prognosis in select patient populations. Van der Schaff et al. [18] reported mortality in STEMI patients with single-vessel disease, MVD, and a CTO of $8 \%$, $16 \%$, and $35 \%$, respectively. CTO is an independent predictor of mortality in patients receiving primary PCI. Bataille et al. [19] also proved that CTO was independently associated with the occurrence of mortality in STEMI patients presenting with cardiogenic shock. Although it is thought that retrograde collateralization provides adequate blood flow to reduce ischemia, prior studies have shown that normal coronary flow reserve could only be achieved in less than $10 \%$ of CTO patients despite well-developed collateral circulation [20]. Sachdeva et al. [21] further reported that all patients with occluded coronary arteries showed an ischemic fractional flow reserve, even with severe regional dysfunction or well-developed collaterals.
Nowadays, PCI is becoming the preferred revascularization method due to the rapid advancement in equipment and techniques as well as a growing expertise among dedicated operators [22-27]. The rate of successful CTO PCI has increased to $90 \%$ in experienced institutions [28]. Several original studies and meta-analyses proved that successful PCI of CTO was related to decreased rates of adverse clinical outcomes, such as mortality, MI, and revascularization. However, CTO recanalization was associated with a much higher risk of complications in comparison with non-CTO interventions, especially perforation. Across multiple contemporary registries, tamponade occurred in $0.4 \%$ to $1.3 \%$ of cases. However, most of the evidence comes from observational research, which inevitably has a lot of potential bias.

To address the potential bias caused by the observational nature of studies comparing successful with failed PCI of CTO, recently, 3 randomized controlled studies compared CTO PCI versus optimal medical therapy in CTO patients; Decision-CTO [29], Euro-CTO [30], and REVASC [31]. Decision-CTO and Euro-CTO showed no advantage of CTO recanalization regarding the composite endpoint MACE, which was inconsistent with the REVASC trial. It is widely established that CTO PCI carries advantages in terms of improving symptoms compared with drug therapy alone except Decision-CTO. These differences could be due to the limitations found in the Decision-CTO trial, such as the slow and early termination of enrollment, the high percentage of cross-over in 


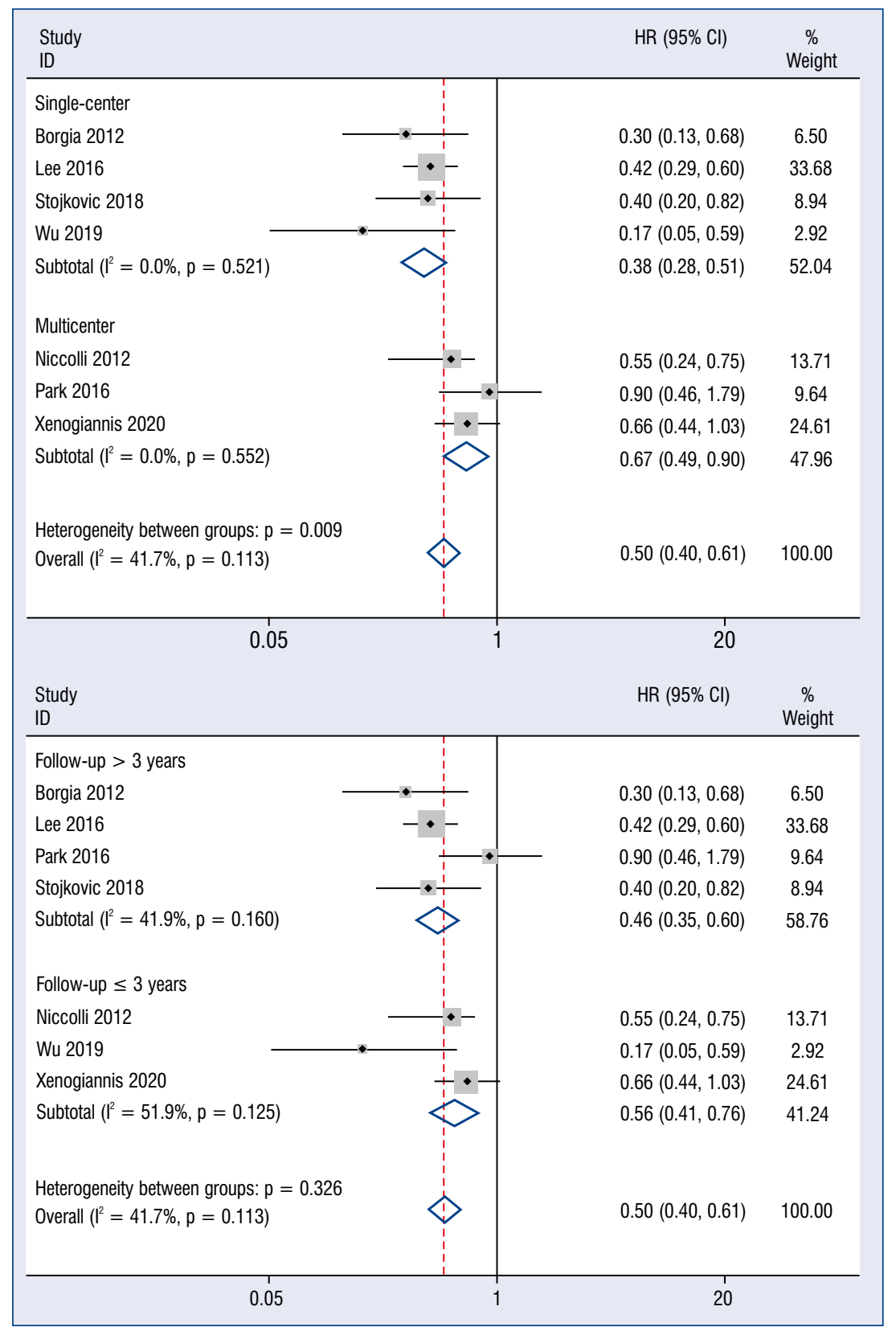

Figure 3. Forest plots for hazard ratios (HRs) of subgroup analyses for successful chronic total occlusion percutaneous coronary intervention; $\mathrm{Cl}$ - confidence interval.

both arms, the high frequency of PCI for non-CTO lesions and the inclusion of patients with mild or absent symptoms. Meta-analysis including these randomized controlled trials (RCTs) and 5 observational studies revealed that CTO recanalization using DES was related to improved cardiac prognosis when compared with optimal medical therapy alone. However, no obvious difference was observed in the RCT subgroup consisting of 1,399 patients [32]. More RCTs are needed to explore the safety and efficacy of CTO PCI.
Current guidelines emphasize the critical role of evaluating viable myocardium in patients presenting with coronary CTO $[8,9]$. The major considerations when selecting individuals who are clinically appropriate and will gain improved prognosis from recanalization of occluded lesions are the presence or absence as well as the extent of myocardial viability. However, myocardial viability assessments are not currently standard processes in real-world clinical diagnosis and treatment. 


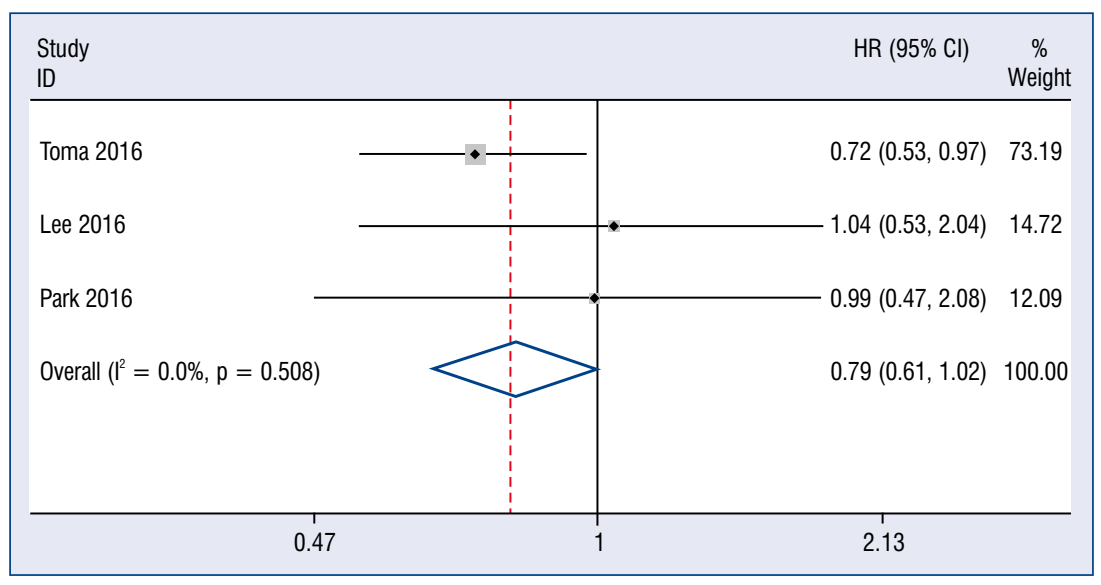

Figure 4. Forest plot for the correlation between successful chronic total occlusion percutaneous coronary intervention and all-cause mortality; $\mathrm{HR}$ - hazard ratio; $\mathrm{Cl}$ - confidence interval.

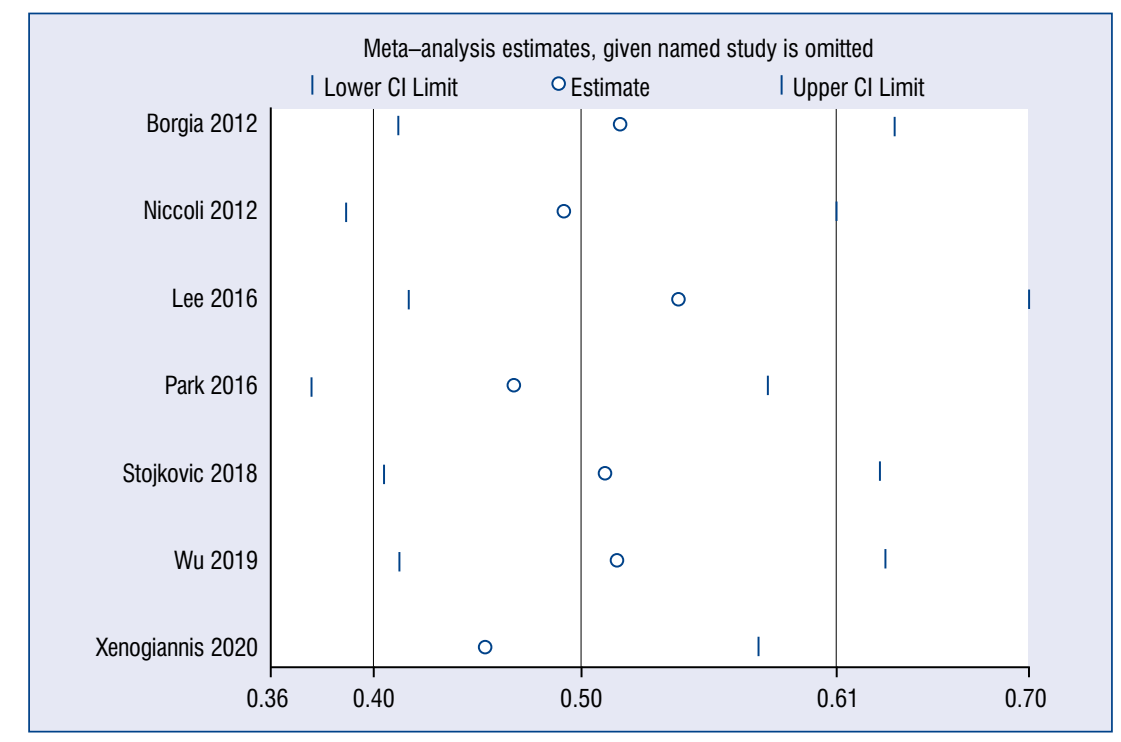

Figure 5. Sensitivity analysis for major adverse cardiac events; $\mathrm{Cl}$ - confidence interval.

Apparently, prior studies have been suboptimally designed and performed. The absence of standardized end points and the discrepancy in definitions also prevent consistency and uniform interpretability of reported results in CTO intervention. CTO-ARC has provided uniform definitions for endpoints specific to CTO interventions and recommends a consensus framework for the design of clinical trials and registries.

Despite considerable retrospective and registry data suggesting a clinical benefit of PCI of a CTO, a clear demonstration of benefit from prospective randomized trials has not been forth- coming. Future trials using uniform definitions for endpoints may change the current landscape.

\section{Limitations of the study}

Since the study objective was to compare successful versus failed operations, the data of the current meta-analysis were obtained from observational trials without exception. The pooled results are affected by confounding factors, although the Newcastle-Ottawa Scale evaluation showed high quality. The baseline data and angiographic features of the two groups were obviously unbalanced, thus, the results could not be extended arbitrarily. In ad- 
dition, post-discharge medication information was not collected in the original studies.

\section{Conclusions}

Despite certain limitations, this analysis showed that successful CTO PCI is associated with improved long-term outcomes. However, the presented data are a comparison between successful and failed PCI of CTO, and any extrapolation of these results to compare PCI and medical treatment should be undertaken with caution. RCTs are needed to further optimize treatment strategies for CTO.

\section{Acknowledgments}

This work was supported by the capital health research and development of special (2018-2-2063).

\section{Conflict of interest: None declared}

\section{References}

1. Ybarra LF, Rinfret S, Brilakis ES, et al. Definitions and Clinical Trial Design Principles for Coronary Artery Chronic Total Occlusion Therapies: CTO-ARC Consensus Recommendations. Circulation. 2021; 143(5): 479-500, doi: 10.1161/CIRCULATIONAHA.120.046754, indexed in Pubmed: 33523728.

2. Christofferson RD, Lehmann KG, Martin GV, et al. Effect of chronic total coronary occlusion on treatment strategy. Am J Cardiol. 2005; 95(9): 1088-1091, doi: 10.1016/j.amjcard.2004.12.065, indexed in Pubmed: 15842978.

3. Fefer P, Knudtson ML, Cheema AN, et al. Current perspectives on coronary chronic total occlusions: the Canadian Multicenter Chronic Total Occlusions Registry. J Am Coll Cardiol. 2012; 59(11): 991-997, doi: 10.1016/j.jacc.2011.12.007, indexed in Pubmed: 22402070 .

4. Jeroudi OM, Alomar ME, Michael TT, et al. Prevalence and management of coronary chronic total occlusions in a tertiary Veterans Affairs hospital. Catheter Cardiovasc Interv. 2014; 84(4): 637-643, doi: 10.1002/ccd.25264, indexed in Pubmed: 24142769.

5. Werner GS, Gitt AK, Zeymer U, et al. Chronic total coronary occlusions in patients with stable angina pectoris: impact on therapy and outcome in present day clinical practice. Clin Res Cardiol. 2009; 98(7): 435-441, doi: 10.1007/s00392-009-0013-5, indexed in Pubmed: 19294443.

6. Tsai TT, Stanislawski MA, Shunk KA, et al. Contemporary incidence, management, and long-term outcomes of percutaneous coronary interventions for chronic coronary artery total occlusions: insights from the VA CART program. JACC Cardiovasc Interv. 2017; 10(9): 866-875, doi: 10.1016/j.jcin.2017.02.044, indexed in Pubmed: 28473108.

7. Claessen BE, Dangas GD, Weisz G, et al. Prognostic impact of a chronic total occlusion in a non-infarct-related artery in patients with ST-segment elevation myocardial infarction: 3-year results from the HORIZONS-AMI trial. Eur Heart J. 2012; 33(6): 768-775, doi: 10.1093/eurheartj/ehr471, indexed in Pubmed: 22240495 .
8. Levine GN, Bates ER, Blankenship JC, et al. 2011 ACCF/ /AHA/SCAI Guideline for Percutaneous Coronary Intervention: a report of the American College of Cardiology Foundation/ American Heart Association Task Force on Practice Guidelines and the Society for Cardiovascular Angiography and Interventions. Circulation. 2011; 124(23): e574-e651, doi: 10.1161/ CIR.0b013e31823ba622, indexed in Pubmed: 22064601.

9. Neumann FJ, Sousa-Uva M, Ahlsson A, et al. 2018 ESC/EACTS Guidelines on myocardial revascularization. Eur Heart J. 2019; 40: 87-165, doi: 10.1093/eurheartj/ehy394., indexed in Pubmed: 30165437.

10. Park JP, Han S, Sung KC, et al. Seven-Year clinical outcomes of successful versus failed revascularization using drug-eluting stents for the treatment of coronary chronic total occlusion. J Invasive Cardiol. 2016; 28(6): 229-236, indexed in Pubmed: 26567453.

11. Borgia F, Viceconte N, Ali O, et al. Improved cardiac survival, freedom from MACE and angina-related quality of life after successful percutaneous recanalization of coronary artery chronic total occlusions. Int J Cardiol. 2012; 161(1): 31-38, doi: 10.1016/j. ijcard.2011.04.023, indexed in Pubmed: 21722979.

12. Niccoli G, De Felice F, Belloni F, et al. Late (3 years) follow-up of successful versus unsuccessful revascularization in chronic total coronary occlusions treated by drug eluting stent. Am J Cardiol. 2012; 110(7): 948-953, doi: 10.1016/j.amjcard.2012.05.025, indexed in Pubmed: 22721573.

13. Toma A, Gick M, Minners J, et al. Survival after percutaneous coronary intervention for chronic total occlusion. Clin Res Cardiol. 2016; 105(11): 921-929, doi: 10.1007/s00392-016-1000-2.

14. Lee PH, Lee SW, Park HS, et al. Successful recanalization of native coronary chronic total occlusion is not associated with improved long-term survival. JACC Cardiovasc Interv. 2016; 9(6): 530-538, doi: 10.1016/j.jcin.2015.11.016, indexed in Pubmed: 26947387.

15. Wu KZ, Huang ZH, Zhong ZA, et al. Successful treatment of complex coronary chronic total occlusions improves midterm outcomes. Ann Transl Med. 2019; 7(9): 194, doi: 10.21037/ atm.2019.05.09, indexed in Pubmed: 31205912.

16. Xenogiannis I, Nikolakopoulos I, Krestyaninov O, et al. Impact of successful chronic total occlusion percutaneous coronary interventions on subsequent clinical outcomes. J Invasive Cardiol. 2020; 32(11): 433-439, indexed in Pubmed: 32568095.

17. Stojkovic S, Juricic S, Dobric M, et al. Improved propensity-score matched long-term clinical outcomes in patients with successful percutaneous coronary interventions of coronary chronic total occlusion. Int Heart J. 2018; 59(4): 719-726, doi: 10.1536/ihj.17360, indexed in Pubmed: 29877305.

18. van der Schaaf RJ, Vis MM, Sjauw KD, et al. Impact of multivessel coronary disease on long-term mortality in patients with ST-elevation myocardial infarction is due to the presence of a chronic total occlusion. Am J Cardiol. 2006; 98(9): 1165-1169, doi: 10.1016/j.amjcard.2006.06.010, indexed in Pubmed: 17056319.

19. Bataille Y, Déry JP, Larose É, et al. Deadly association of cardiogenic shock and chronic total occlusion in acute ST-elevation myocardial infarction. Am Heart J. 2012; 164(4): 509-515, doi: 10.1016/j.ahj.2012.07.008, indexed in Pubmed: 23067908.

20. Werner GS, Surber R, Ferrari M, et al. The functional reserve of collaterals supplying long-term chronic total coronary occlusions in patients without prior myocardial infarction. Eur Heart J. 2006; 27(20): 2406-2412, doi: 10.1093/eurheartj/ehl270, indexed in Pubmed: 17003048. 
21. Sachdeva R, Agrawal M, Flynn SE, et al. The myocardium supplied by a chronic total occlusion is a persistently ischemic zone. Catheter Cardiovasc Interv. 2014; 83(1): 9-16, doi: 10.1002/ ccd.25001, indexed in Pubmed: 23703867.

22. Gutiérrez-Chico JL, Cortés C, Ayoub M, et al. Subintimal shift as mechanism for side-branch occlusion in percutaneous treatment of chronic total occlusions with bifurcation lesions. Cardiol J. 2021 [Epub ahead of print], doi: 10.5603/CJ.a2021.0079, indexed in Pubmed: 34231874.

23. Chu M, Martínez-Hervás-Alonso MÁ, Reisbeck B, et al. The yin-yang sign in the detection of subintimal hematoma with highdefinition intravascular ultrasound. Cardiol J. 2020; 27(1): 81-82, doi: 10.5603/CJ.2020.0015, indexed in Pubmed: 32103478.

24. Mashayekhi K, Behnes M. The role of intravascular ultrasound in the treatment of chronic total occlusion with percutaneous coronary intervention. Cardiol J. 2020; 27(1): 4-5, doi: 10.5603/ CJ.2020.0013, indexed in Pubmed: 32103475.

25. Guelker JE, Bufe A, Blockhaus C, et al. The atherogenic index of plasma and its impact on recanalization of chronic total occlusion. Cardiol J. 2020; 27(6): 756-761, doi: 10.5603/CJ.a2018.0064, indexed in Pubmed: 29924378.

26. Guelker JE, Blockhaus C, Bufe A, et al. In-hospital outcome of re-attempted percutaneous coronary interventions for chronic total occlusion. Cardiol J. 2021 [Epub ahead of print], doi: 10.5603/CJ.a2021.0012, indexed in Pubmed: 33634842.

27. Zhao Y, Peng H, Li X, et al. The impact of dissection and re-entry versus wire escalation techniques on long-term clinical out- comes in patients with chronic total occlusion lesions following percutaneous coronary intervention: An updated meta-analysis. Cardiol J. 2021; 28(3): 369-383, doi: 10.5603/CJ.a2020.0026, indexed in Pubmed: 32104900.

28. Shah A. Chronic total occlusion coronary intervention: in search of a definitive benefit. Methodist Debakey Cardiovasc J. 2018; 14(1): 50-59, doi: 10.14797/mdcj-14-1-50, indexed in Pubmed: 29623172.

29. Lee SW, Lee PH, Ahn JM, et al. Randomized trial evaluating percutaneous coronary intervention for the treatment of chronic total occlusion. Circulation. 2019; 139(14): 1674-1683, doi: 10.1161/ CIRCULATIONAHA.118.031313, indexed in Pubmed: 30813758.

30. Werner GS, Martin-Yuste V, Hildick-Smith D, et al. A randomized multicentre trial to compare revascularization with optimal medical therapy for the treatment of chronic total coronary occlusions. Eur Heart J. 2018; 39(26): 2484-2493, doi: 10.1093/eurheartj/ ehy220, indexed in Pubmed: 29722796.

31. Mashayekhi K, Nührenberg TG, Toma A, et al. A randomized trial to assess regional left ventricular function after stent implantation in chronic total occlusion: the REVASC trial. JACC Cardiovasc Interv. 2018; 11(19): 1982-1991, doi: 10.1016/j. jcin.2018.05.041, indexed in Pubmed: 30219327.

32. Abo-Aly M, Misumida N, Backer N, et al. Percutaneous coronary intervention with drug-eluting stent versus optimal medical therapy for chronic total occlusion: systematic review and meta-analysis. Angiology. 2019; 70(10): 908-915, doi: 10.1177/0003319719858823, indexed in Pubmed: 31256614. 